\title{
Voxel-Based Morphometry in Tau-Positive and Tau-Negative Frontotemporal Lobar Degenerations
}

\author{
Jennifer L. Whitwella Jason D. Warren ${ }^{a}$ Keith A. Josephs ${ }^{a, c}$ \\ Alison K. Godbolt ${ }^{a}$ Tamas Revesz ${ }^{b}$ Nick C. Fox ${ }^{a}$ Martin N. Rossor ${ }^{a}$ \\ a Dementia Research Centre and bepartment of Molecular Neuroscience, Institute of Neurology, London, UK; \\ 'Department of Neurology, Mayo Clinic, Rochester, Minn., USA
}

\section{Key Words}

Tau · Frontotemporal lobar degeneration .

Voxel-based morphometry · Magnetic resonance imaging

\begin{abstract}
Background: The identification of specific, diagnostically useful predictors of protein dysfunction in the frontotemporal lobar degenerations (FTLD) is a problem of great clinical and biological interest. Correlations between regional patterns of tissue loss and specific proteinopathies have not been established. Objective: Specific brain imaging correlates of protein tau dysfunction were sought using voxel-based morphometry in FTLD subgroups with and without tau pathology. Methods: Seventeen patients with pathologically or genetically confirmed diagnoses of FTLD who had undergone volumetric brain magnetic resonance imaging (MRI) were identified retrospectively and tau-positive $(n=9)$ and tau-negative $(n=8)$ subgroups were defined. MRI data were compared with healthy age- and sex-matched controls using voxel-based morphometry implemented in a statistical parametric mapping software package. Results: Compared with controls, tau-positive and tau-negative sub-
\end{abstract}

\section{KARGER}

Fax + 41613061234

E-Mail karger@karger.ch

www. karger.com
(C) 2004 S. Karger AG, Basel

$1660-2854 / 04 / 0015-0225 \$ 21.00 / 0$

Accessible online at:

www. karger.com/ndd groups had extensive common areas of regional brain atrophy predominantly affecting the frontal and anterior temporal lobes. No specific brain imaging features were identified for either subgroup. Conclusion: Patterns of frontotemporal atrophy do not predict the presence or absence of tau pathology; conversely, different immunohistochemical profiles are associated with similar patterns of regional vulnerability to neuronal loss in FTLD.

Copyright $\odot 2004$ S. Karger AG, Basel

As our understanding of the molecular basis of the degenerative dementias improves, the correlation of specific patterns of tissue loss with disordered protein function in these diseases holds considerable clinical and biological interest [1-5]. The advent of volumetric brain magnetic resonance imaging (MRI) has enabled the identification of distinctive patterns of regional atrophy in a number of clinical dementia syndromes and such patterns can reliably distinguish Alzheimer from non-Alzheimer dementias [6-11]. However, the substantial clinical and genetic heterogeneity within the non-Alzheimer group has so far precluded the identification of specific radiological correlates of protein dysfunction in these diseases [1-3, 11]. This problem is particularly relevant in the large
Prof. M.N. Rossor

Dementia Research Centre

Institute of Neurology

Queen Square, London WC1N 3BG (UK)

Tel. +44 207829 8773, Fax +44 207676 2066, E-Mail m.rossor@dementia.ion.ucl.ac.uk 
group of frontotemporal lobar degenerations (FTLD), in which attempts to identify specific clinical and radiological phenotypes of protein pathophysiology have been largely unsuccessful [1-3]. Here we describe a detailed analysis of MRI features in a population of individuals with pathologically and genetically defined FTLD: we sought to identify specific patterns of regional brain atrophy in individuals with and without tau pathology. Tau is a microtubule-associated protein that has been implicated in the pathogenesis of many sporadic and hereditary neurodegenerative diseases and a number of mutations in the tau gene on chromosome 17 have been identified [1-5]. The tauopathies are a clinically, radiologically and pathologically diverse group of diseases. Although postmortem studies have not established macroscopic patterns of brain involvement that reliably distinguish diseases with and without tau pathology [12, 13], pathological case series are likely collectively to represent a more advanced stage of disease; brain imaging during life might detect specific macroscopic correlates of tau dysfunction at an earlier stage of disease. In the present study, the MRI phenotypes of tau-positive and tau-negative patients were compared using voxel-based morphometry: an unbiased statistical methodology that is emerging as a promising tool in the identification of characteristic patterns of brain atrophy in a number of the degenerative dementias [10, 14].

\section{Methods}

\section{Subjects}

Seventeen patients with pathologically or genetically confirmed diagnoses of FTLD who had undergone volumetric brain MRI were identified retrospectively from referrals to the Dementia Research Group at the Institute of Neurology, London. Patients were diagnosed clinically as having frontotemporal dementia (13 cases), semantic dementia (3 cases) or progressive non-fluent aphasia (PNFA; 1 case) according to current consensus criteria [15].

\section{Pathological and Genetic Data}

The pathological diagnosis was established either at postmortem or on antemortem brain biopsy; 1 patient had an identified tau mutation and a typical clinical presentation without pathological confirmation. After formalin fixation and paraffin embedding, tissue blocks were examined using routine techniques including haematoxylin and eosin, Luxol fast blue, cresyl violet and modified Bielschowsky's silver stains. All specimens were immunostained with antibodies against tau (tau 12E8; Elan Pharmaceuticals) and ubiquitin (DAKO, Carpinteria, Calif., USA). The pathological diagnosis was based on consensus criteria [16]. In patients with tau pathology and an autosomal dominant pedigree, the tau gene was sequenced and screened for known mutations to identify cases with frontotemporal dementia-parkinsonism linked to chromosome 17 (FTDP-17).
Table 1. Characteristics of tau-positive and tau-negative subgroups in this study

\begin{tabular}{lllll}
\hline & $\begin{array}{l}\text { Age } \\
\text { years }\end{array}$ & $\begin{array}{l}\text { Duration of } \\
\text { symptoms } \\
\text { years }\end{array}$ & $\begin{array}{l}\text { MMSE } \\
\text { score }\end{array}$ & $\begin{array}{l}\text { CDR } \\
\text { score }\end{array}$ \\
\hline Tau-positive $(\mathrm{n}=9)$ & $52(8.7)$ & $4(2.1)$ & $21(4.5)$ & $1.4(0.2)$ \\
Tau-negative $(\mathrm{n}=8)$ & $62(6.8)$ & $4(2.3)$ & $22(5.5)$ & $1.2(0.5)$
\end{tabular}

Values refer to mean and standard deviation in parentheses at time of brain MRI. CDR = Clinical Dementia Rating scale; $\mathrm{MMSE}=$ Mini-Mental State Examination score.

Two pathological subgroups were defined: patients with tau inclusions ( 4 cases of FTDP-17, 5 cases of sporadic Pick's disease) constituted the 'tau-positive' subgroup; patients with ubiquitin-positive, tau-negative inclusions ( 8 cases) constituted the 'tau-negative' subgroup. Characteristics of the tau-positive and tau-negative subgroups are summarized in table 1 . Overall, both subgroups were wellmatched with respect to disease duration and clinical severity (as indexed by Mini-Mental State Examination score and Clinical Dementia Rating scale); the mean age of patients in the tau-negative subgroup was 10 years older than in the tau-positive subgroup.

\section{MRI Acquisition}

In all patients and in 20 healthy age- and sex-matched controls, T1-weighted volumetric MR scans were acquired using a 1.5-tesla Signa unit (General Electric Medical Systems, Milwaukee, Wisc., USA) with a spoiled gradient-echo technique. Imaging parameters were as follows: time to echo $5 \mathrm{~ms}$, time to repeat $35 \mathrm{~ms}$, flip angle $35^{\circ}$, and field of view $24 \times 24 \times 19.2 \mathrm{~cm}$. This sequence yielded 124 contiguous 1.5 -mm-thick coronal slices.

\section{Image Analysis}

Distributions of brain atrophy in the tau-positive and tau-negative groups were compared with controls using statistical parametric mapping software SPM99 (Wellcome Department of Imaging Neuroscience, London, UK; http//:www.fil.ion.ucl.ac.uk/spm) under MATLAB $6{ }^{\circledR}$ (Mathworks, Sherbourn, Mass., USA); the various processing steps have been described in detail previously [10]. All images were first normalized to a customized template. This customized template was based on a mean brain image derived from a group of 10 normal controls and 10 patients with a neurodegenerative disease (5 Alzheimer's disease, 5 FTLD), all age- and gender-matched to the study cohort and acquired on the same MR scanner; the mean image was normalized to a standard stereotactic space defined by the Montreal Neurological Institute (MNI) standard brain [17] and spatially smoothed using an isotropic Gaussian kernel of $8 \mathrm{~mm}$ full width at half maximum (FWHM). Each subject's normalized brain image was segmented into grey matter, white matter and cerebrospinal fluid and each grey matter image was masked to exclude all non-brain voxels with a brain region performed using a semi-automated, iterative 3dimensional morphologic technique implemented in MIDAS image analysis software [18]. Masked grey matter images were modulated 


\section{Tau positive Tau negative}

Fig. 1. Group statistical parametric maps (SPMs) of significant volume loss in tau-positive and tau-negative subgroups compared with age-matched healthy controls. SPMs have been rendered on coronal and axial sections of the customized template MR brain volume (see Methods) and thresholded at an uncorrected voxel significance level of $p<$ 0.0001 for display purposes; the $\mathrm{Z}$ score at each voxel is coded according to the colour scale (right). In both tau-positive and taunegative cases, atrophy involves extensive bilateral frontal and anterior temporal areas. No regions are involved specifically in the tau-positive or in the tau-negative subgroups, although there are qualitative differences in the profile of temporal lobe atrophy between the two groups (see text).

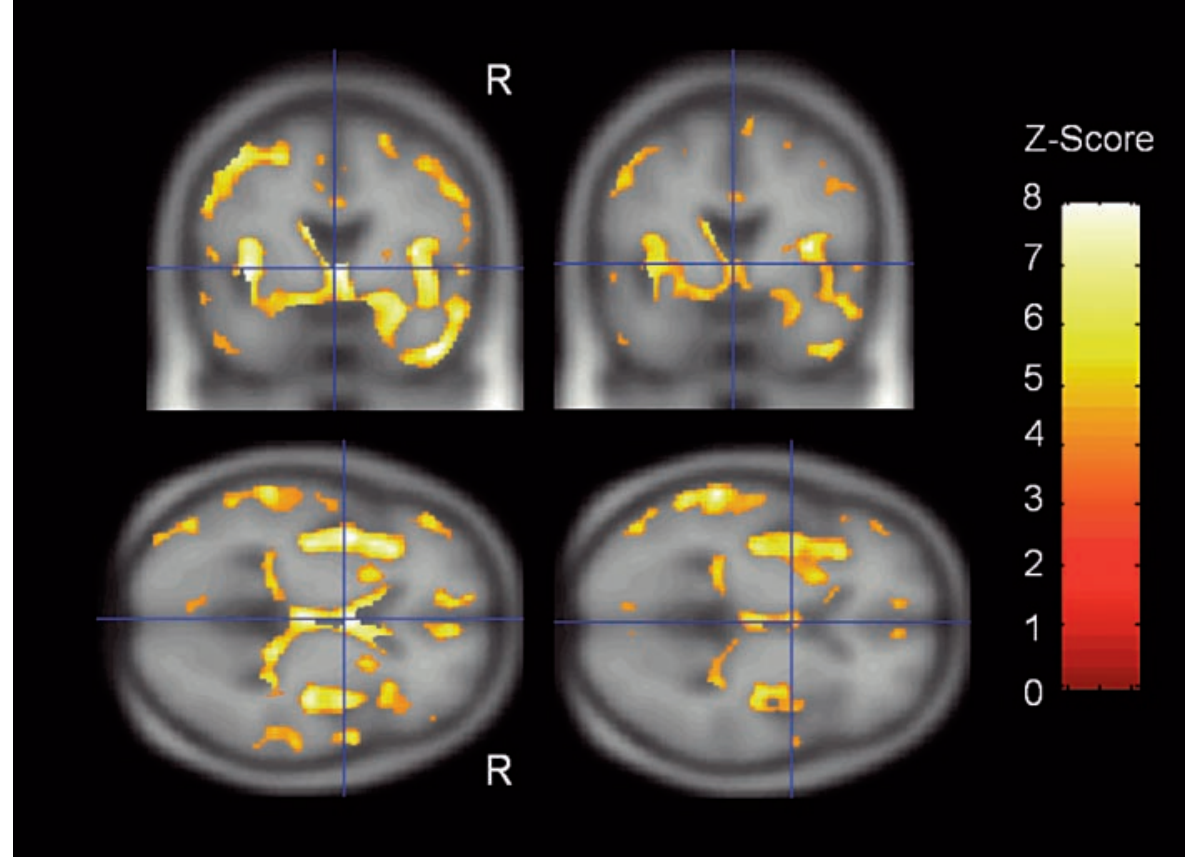

and smoothed with an isotropic Gaussian kernel of $8 \mathrm{~mm}$ FWHM [19]. Regionally specific differences in grey matter volume between the tau-positive and tau-negative subgroups and between each pathological subgroup and controls were assessed using a single subject condition and covariate model implemented in statistical parametric mapping (SPM99) software (http//:www.fil.ion.ucl.ac.uk/spm); age and gender were included in the model as nuisance variables. Volume changes in the tau-positive and tau-negative subgroups relative to the control group and between the two pathological subgroups were assessed at every brain voxel by estimating the $t$ score at a significance threshold of $\mathrm{p}<0.05$ corrected for multiple comparisons across the entire brain volume according to Gaussian random field theory [19].

In every subject, the whole-brain volume and total intracranial volume (TIV) were estimated from the raw T1-weighted MRI volume using MIDAS image analysis software as previously described $[18,20]$. To estimate whole-brain volume, every axial slice between the lowest point of the cerebellum and the superior point of the cortex was measured using a fixed CSF-brain intensity threshold (60\% of mean brain signal intensity). To estimate TIV, each volume was registered to the standard MNI template and every tenth axial slice was measured using a fixed threshold $(30 \%$ of mean brain signal intensity) to outline the outer border of dura with linear interpolation between slices. Segmentations were manually checked and edited to ensure accuracy. Whole-brain volumes were normalized to TIV and the mean normalized whole-brain volumes in the tau-positive and tau-negative groups were compared in a one-way analysis of variance.

VBM and FTLD Immunohistochemistry

\section{Results}

Both the pathological subgroups had significant and extensive cortical volume loss compared with healthy controls (fig. 1): in both the tau-positive and tau-negative cases, atrophy involved predominantly frontal and temporal cortices and was somewhat more pronounced in the right hemisphere. Areas of atrophy that were common to both tau-positive and tau-negative cases clustered in the anterior temporal lobes, frontal opercula and frontal convexities bilaterally. The locations of maximal volume loss within each of these brain regions in the tau-positive and tau-negative subgroups are presented in table 2 . No brain regions were involved exclusively in the tau-positive or tau-negative subgroup. However, inspection of figure 1 and table 2 suggests differences in the distribution of atrophy within the common affected regions between the taupositive and tau-negative subgroups: for example, atrophy was relatively more severe in the right anterior temporal lobe and bilateral prefrontal regions in the tau-positive cases, and relatively more severe in the left posterior temporal lobe in the tau-negative cases. A direct comparison of the tau-positive and tau-negative cases yielded no 
Table 2. Locations of maximal volume loss in tau-positive and tau-negative cases

\begin{tabular}{llrrrr}
\hline Region & Side & \multicolumn{3}{c}{ Coordinates of maximal volume loss, $\mathrm{mm}$} & \multirow{2}{*}{ Z score } \\
\cline { 5 - 6 } & & $\mathrm{x}$ & $\mathrm{y}$ & $\mathrm{z}$ & \\
\hline Tau-positive & & & & & \\
Premotor & $\mathrm{R}$ & 57 & -9 & 38 & 6.7 \\
Inferior anterior temporal & $\mathrm{R}$ & 44 & 7 & -42 & 6.6 \\
Orbitofrontal & $\mathrm{R}$ & 6 & 4 & -9 & 6.5 \\
Frontal operculum & $\mathrm{L}$ & -42 & 3 & -3 & 6.2 \\
Frontal operculum & $\mathrm{R}$ & 37 & -2 & 9 & 6.1 \\
Medial frontal & $\mathrm{R}$ & 5 & 25 & 44 & 6.1 \\
Lateral anterior temporal & $\mathrm{R}$ & 54 & 8 & -25 & 5.9 \\
Hippocampus & $\mathrm{R}$ & 34 & -13 & -16 & 5.8 \\
Postcentral gyrus & $\mathrm{L}$ & -60 & -24 & 37 & 5.8 \\
Orbitofrontal & $\mathrm{L}$ & -39 & 34 & -13 & 5.8 \\
\hline Tau-negative & & & & & \\
Frontal operculum & $\mathrm{R}$ & 36 & -1 & 9 & 6.3 \\
Posteriorsuperior temporal & $\mathrm{L}$ & -63 & -37 & 0 & 5.9 \\
Postcentral & $\mathrm{L}$ & -58 & -55 & 33 & 5.9 \\
\hline
\end{tabular}

Data are derived from comparisons of each of the two FTLD subgroups with the healthy control subjects. Local maxima are shown for both the tau-positive and tau-negative subgroups contrasted with controls. Voxel coordinates are in millimetres after transformation into standard MNI stereotactic space [17]. Only those local maxima exceeding a stringent statistical threshold of $\mathrm{p}<0.001$ corrected for multiple comparisons across the entire brain volume are shown. significant differences in regional grey matter volume between the two subgroups (threshold $\mathrm{p}<0.05$ corrected). Mean TIV-normalized whole-brain volumes did not differ significantly between the tau-positive and tau-negative subgroups (threshold $\mathrm{p}<0.05$ ).

\section{Discussion}

This study suggests that patterns of frontotemporal atrophy do not predict the presence or absence of tau pathology, and conversely, that different immunohistochemical profiles are associated with similar patterns of regional vulnerability to neuronal loss in FTLD. These findings support the view that the topography of atrophy rather than immunohistochemistry determines phenotype in FTLD [3], and emphasize the pathological and genetic heterogeneity of these diseases. The results are consistent with pathological case series that have failed to identify specific macroscopic correlates of tau pathology $[12,13]$. However, the apparent lack of correlation between tau pathology and the distribution of atrophy on brain imaging should be interpreted cautiously. The num- ber of patients in each of the pathological subgroups here was relatively small, and the study may, therefore, have lacked sensitivity to detect subtle differences in the crosssectional profile of atrophy that might emerge in larger populations. The present data (fig. 1, table 2) do suggest differences in the profile of frontotemporal atrophy between tau-positive and tau-negative subgroups; however, these differences did not attain statistical significance. Even if such differences are substantiated in larger cohorts, they are unlikely to be useful in predicting the pathological diagnosis in the individual patient with an established clinical FTLD syndrome. All of the patients in this study fulfilled consensus clinical criteria for the diagnosis of FTLD [15]; it remains possible that pathologically determined differences in the profile of frontotemporal atrophy may be present earlier in the course of these diseases (possibly preceding the onset of clinical symptoms), with convergent involvement of cortical regions as the pathological process becomes more widely distributed. In Alzheimer's disease, it is known that the pattern of regional atrophy may shift with advancing disease [14, 21]; however, little information is presently available concerning the evolution of atrophy in FTLD. The prospect 
of disease-modifying therapies in the neurodegenerative diseases [1] means that the detection of clinicoradiological correlations would have both maximal specificity and maximal clinical value early in the course of the disease.

It is important to keep in mind that FTLD is highly heterogeneous clinically as well as pathologically, and certain clinical phenotypes such as PNFA are underrepresented in the present sample: specific radiological and pathological correlations may exist for clinically defined subpopulations within the FTLD spectrum, though not for the group considered collectively. In addition, an uncertain proportion of cases classified as tau-negative using currently available pathological and genetic criteria may nevertheless have tau dysfunction, which might produce a similar pattern of tissue destruction [1]. The rightward asymmetric distribution of anterior temporal lobe involvement in both subgroups here might appear somewhat at variance with previous studies describing a leftward asymmetry of temporal atrophy $[6,8,22]$ : this difference may reflect the relative preponderance of behavioural and personality disturbance (the frontotemporal dementia syndrome) in the majority (13/17) of cases in the present cohort. In contrast to primary impairments of language (PNFA) or semantic memory (semantic dementia), which are associated with selective involvement of frontotemporal regions in the dominant hemisphere [6, 11], frontal behavioural presentations are frequently associated with non-dominant frontotemporal atrophy [2325].
It remains possible that differences between pathological subgroups may be detected using imaging parameters other than the cross-sectional extent or severity of volume loss: for example, different rates of global or regional brain atrophy [26]. The present cross-sectional study detected no significant differences in whole-brain volume between tau-positive and tau-negative cases after a similar duration of clinical disease. However, the direct demonstration of differential rates of atrophy would require the prospective analysis of serial volumetric MR images registered into a common stereotactic space; moreover, differential rates of atrophy might manifest at the level of particular brain regions rather than globally [14]. We therefore suggest that the present findings underline the need for further prospective studies of genetically defined 'at risk' FTLD populations.

\section{Acknowledgments}

The authors thank the subjects and their families for participation, Dr. Hilary Watt for statistical advice and Dr. Peter Lantos for the initial neuropathological assessment of a number of cases. This work was supported by the European Union under the programme 'Quality of Life and Management of Living Resources', Key Action 3 'The Cell Factory', Contract No. QLK3-CT-2001-02362. J.L. Whitwell receives financial support from the Special Trustees of UCLH/ NHNN. N.C. Fox holds a Medical Research Council Senior Clinical Fellowship. K.A. Josephs was a Mayo Foundation Scholar to the Dementia Research Centre and the Queen Square Brain Bank Department of Molecular Neurosciences, Institute of Neurology, UCL, London, UK.

\section{References}

1 Rosso SM, Van Swieten JC: New developments in frontotemporal dementia and parkinsonism linked to chromosome 17. Curr Opin Neurol 2002; 15:423-428.

$\checkmark 2$ Cummings JL: Toward a molecular neuropsychiatry of neurodegenerative diseases. Ann Neurol 2003;54:147-154.

-3 Petersen RC: Focal dementia syndromes: In search of the gold standard. Ann Neurol 2001; 49:421-423.

$\checkmark 4$ Hutton M, Lendon CL, Rizza P, Baker M, Froelich S, Houlden H, Pickering-Brown S, Chakraverty S, Isaacs A, Grover A, Hackett J, Adamson J, Lincoln S, Dickson D, Davies P, Petersen RC, Stevens M, de-Graaff E, Wauters E, van-Baren J, Hillebrand M, Joosse M, Kwon JM, Nowotny P, Heutink P: Association of missense and $5^{\prime}$ splice site mutations in tau with the inherited dementia FTDP-17. Nature 1998:393:702-705.

5 Schott JM, Fox NC, Rossor MN: Genetics of the dementias. J Neurol Neurosurg Psychiatry 2002;73(suppl II):27-31.
6 Chan D, Fox NC, Scahill RI, Crum WR, Whitwell JL, Leschziner G, Rossor AM, Stevens JM, Cipolotti L, Rossor MN: Patterns of temporal lobe atrophy in semantic dementia and Alzheimer's disease. Ann Neurol 2001;49:433442.

7 Boxer A, Rankin K, Miller B, Schuff N, Weiner M, Gorno-Tempini ML, Rosen HJ: Cinguloparietal atrophy distinguishes Alzheimer disease from semantic dementia. Arch Neurol 2003;60:949-956.

$>8$ Galton CJ, Patterson K, Graham KS, LambonRalph MA, Williams G, Antoun N, Sakakian BJ, Hodges JR: Differing patterns of temporal atrophy in Alzheimer's disease and semantic dementia. Neurology 2001;57:216-225.

9 Rosen HJ, Gorno-Tempini ML, Goldman WP, Perry RJ, Schuff N, Weiner M, Feiwell R, Kramer JH, Miller BL: Patterns of brain atrophy in frontotemporal dementia and semantic dementia. Neurology 2002;58:198-208.
0 Good CD, Scahill RI, Fox NC, Ashburner J, Friston KJ, Chan D, Crun WR, Rossor MN, Frackowiak RS: Automatic differentiation of anatomical patterns in the human brain: Validation with studies of the degenerative dementias. Neuroimage 2002;17:29-46.

11 Gorno-Tempini ML, Dronkers NF, Rankin KP, Ogar JM, Phengrasamy L, Rosen HJ, Johnson JK, Weinder MW, Miller BL: Cognition and anatomy in three variants of primary progressive aphasia. Ann Neurol 2004;55:335346.

12 Mann DMA, South PW: The topographic distribution of brain atrophy in frontal lobe dementia. Acta Neuropathol 1993;85:334-340.

$\checkmark 13$ Broe M, Hodges JR, Schofield E, Shepherd CE, Kril JJ, Halliday GM: Staging disease severity in pathologically confirmed cases of frontotemporal dementia. Neurology 2003;60:10051011. 
14 Scahill RI, Schott JM, Stevens JM, Rossor MN, Fox NC: Mapping the evolution of regional atrophy in Alzheimer's disease: Unbiased analysis of fluid-registered serial MRI. Proc Natl Acad Sci USA 2002;99:4703-4707.

15 Neary D, Snowden JS, Gustafson L, Passant U, Struss D, Black S, Freedman M, Kertesz A, Robert PH, Albert M, Boone K, Miller BL, Cummings J, Benson DF: Frontotemporal lobar degeneration. A consensus on clinical diagnostic criteria. Neurology 1998;51:1546-1554.

16 McKhann GM, Albert MS, Grossman M, Miller B, Dickson D, Trojanowski J: Clinical and pathological diagnosis of frontotemporal dementia. Arch Neurol 2001;58:1803-1809.

$>17$ Evans AC, Collins DL, Mills SR, Brown ED, Kelly RL, Peters TM: 3D statistical neuroanatomical models from 305 MRI volumes. Proc IEEE Nucl Sci Symp Med Imag Conf 1993;3. 1813-1817.
18 Freeborough PA, Fox NC, Kitney RI: Interactive algorithms for the segmentation and quantitation of 3-D MRI brain scans. Comput Methods Programs Biomed 1997;53:15-25.

$\checkmark 19$ Friston KJ, Holmes AP, Worsley KJ, Poline JP, Frith CD, Frackowiak RSJ: Statistical parametric maps in functional imaging: A general linear approach. Hum Brain Mapp 1995;2: 189-210.

20 Whitwell JL, Crum WR, Watt HC, Fox NC: Normalization of cerebral volumes by use of intracranial volume: Implications for longitudinal quantitative MR imaging. AJNR Am J Neuroradiol 2001;22:1483-1489.

21 Braak H, Braak E: Evolution of the neuropathology of Alzheimer's disease. Acta Neurol Scand Suppl 1996;165:3-12.

22 Boccardi M, Laakso MP, Bresciani L, Galluzi S, Geroldi C, Beltramello A, Soininen H, Frisoni GB: The MRI pattern of frontal and temporal brain atrophy in fronto-temporal dementia. Neurobiol Aging 2003;24:95-103.
23 Rosen HJ, Perry RJ, Murphy J, Kramer JH, Mychack P, Schuff N, Weiner M, Levenson RW, Miller BL: Emotion comprehension in the temporal variant of frontotemporal dementia. Brain 2002;125:2286-2295.

24 Perry RJ, Rosen HR, Kramer JH, Beer JS, Levenson RL, Miller BL: Hemispheric dominance for emotions, empathy and social behaviour: Evidence from right and left handers with frontotemporal dementia. Neurocase 2001;7: 145-160.

25 Liu W, Miller BL, Kramer JH, Rankin K, Wyss-Coray C, Gearhart R, Phengrasamy L, Weiner M, Rosen HJ: Behavioral disorders in the frontal and temporal variants of frontotemporal dementia. Neurology 2004;62:742-748.

-26 Fox NC, Schott JM: Imaging cerebral atrophy: Normal ageing to Alzheimer's disease. Lancet 2004;363:392-394. 